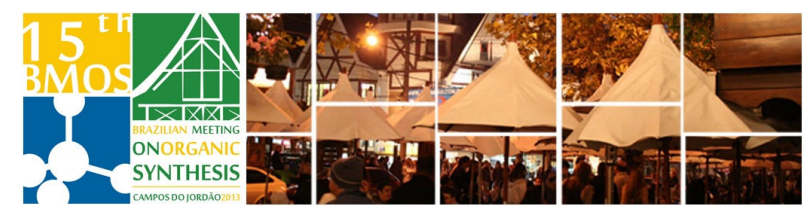

\title{
Benzocarbazolquinones and Benzonaphthofurandiones by Palladium Catalyzed Oxidative C-H Functionalization
}

\author{
Cinthia da Silva Lisboa*, Gustavo dos Santos, Nanci C. de Lucas, Simon J. Garden \\ Instituto de Química, Universidade Federal do Rio de Janeiro, CT, Bloco A, Cidade Universitária - IIha do \\ Fundão, 21949-900, Rio de Janeiro - RJ, BRASIL. \\ *e-mail corresponding author: cinthia lisboa@yahoo.com.br and garden@iq.ufri.br
}

Keywords: naphthoquinone; palladium acetate; $\mathrm{C}-\mathrm{H}$ functionalization

\section{INTRODUCTION}

Quinones are very interesting compounds not only because of their bright colours but also because of their diverse biological properties for example in the treatment of: tuberculosis, malaria, bacterial and parasitic infections and as antineoplastics.

Within a program investigating $\mathrm{C}-\mathrm{H}$ functionalization reactions we recently described improved methodology for the oxidative coupling of anilines with naphthoquinone. ${ }^{2}$ Additionally, we have now studied the oxidative cyclization (formation of a $\mathrm{C}-\mathrm{C}$ bond from two $\mathrm{C}-\mathrm{H}$ bonds) of 2-anilino- and 2phenyloxy- naphthoquinones.

\section{RESULTS AND DISCUSSION}

There are a number of precedents in the literature for the oxidative cyclization of 2anilinonaphthoquinones to benzocarbazolquinones. ${ }^{3}$ In the present study, we have developed upon the method reported by the group of Fagnou (scheme 1). ${ }^{3 \mathrm{~d}}$ We have investigated the use of other cooxidants (such as $\mathrm{Cu}(\mathrm{OAc})_{2} \cdot \mathrm{H}_{2} \mathrm{O}$ ) and the effect of temperature upon the reaction (Table 1 ).<smiles>O=C1C=C(Nc2ccccc2)C(=O)c2ccccc21</smiles>
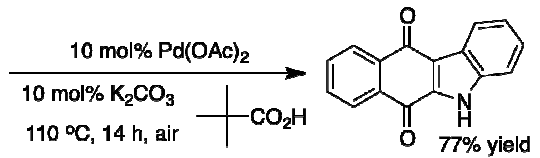

Scheme 1. Synthesis of benzocarbazolquinone. ${ }^{3 \mathrm{~d}}$

Table 1. Reaction conditions (Reaction time: 210 minutes)

\begin{tabular}{|c|c|c|c|c|c|}
\hline Reac. & $\mathrm{Pd}(\mathrm{OAc})_{2}{ }^{\mathbf{a}}$ & $\mathrm{Cu}(\mathrm{OAc})_{2}{ }^{\mathbf{a}}$ & $\mathrm{K}_{2} \mathrm{CO}_{3}{ }^{\mathbf{a}}$ & $\begin{array}{c}\text { Temp. } \\
\left({ }^{\circ} \mathrm{C}\right)\end{array}$ & $\begin{array}{c}\text { Yield } \\
(\%)\end{array}$ \\
\hline 1 & 10 & --- & 10 & 110 & $71^{\mathbf{b}}$ \\
\hline 2 & 10 & 20 & 15 & 110 & $92^{\mathbf{b}}$ \\
\hline 3 & 10 & 20 & 20 & 120 & 63 \\
\hline 4 & 10 & 20 & 20 & 160 & 78 \\
\hline 5 & 10 & 20 & 25 & 180 & 75 \\
\hline 6 & 10 & --- & 25 & 160 & 52 \\
\hline 7 & --- & 20 & 20 & 160 & 0 \\
\hline 8 & 10 & 20 & --- & 160 & 15 \\
\hline
\end{tabular}

${ }^{\mathbf{a}}$ Quantities in mol \%. ${ }^{\mathbf{b}} 19$ hour reaction time.

Initially we reproduced the results of Fagnou and subsequently found that the inclusion of $\mathrm{Cu}(\mathrm{OAc})_{2} \cdot \mathrm{H}_{2} \mathrm{O}$ had a beneficial effect upon the reaction yield (Table 1 , entries 1 and 2). At a shorter reaction time (210 $\mathrm{min}$.) a lesser yield was obtained (entry 3 ). Increasing the reaction temperature made a substantial improvement to the yield whilst maintaining the shorter reaction time (entries 4 and $5)$. Control experiments revealed the importance of the individual components (entries 6, 7 and 8). The methodology was applied to other anilino-and phenyloxy- naphthoquinones (table 2). Regioselective cyclization was observed (entries 3 and 4).

Table 2. Tetracyclic quinones prepared by oxidative $\mathrm{C}-\mathrm{H}$ functionalization.<smiles>[Y][X]c1c([R])c([R])c([R])c2c1C1C(=O)c3ccccc3C(=O)C1=C2[R]</smiles>

\begin{tabular}{|c|c|c|c|}
\hline & Quinone & $\begin{array}{c}\text { Reac. time } \\
(\text { min })\end{array}$ & Yield (\%) \\
\hline 1 & $\mathrm{X}=\mathrm{N} ; \mathrm{R}_{3}=\mathrm{CN}$ & 240 & 78 \\
\hline 2 & $\mathrm{X}=\mathrm{N} ; \mathrm{R}_{1}=\mathrm{OCH}_{3}$ & 340 & 71 \\
\hline 3 & $\mathrm{X}=\mathrm{N} ; \mathrm{R}_{4}=\mathrm{OCH}_{3}$ & 180 & 69 \\
\hline 4 & $\mathrm{X}=\mathrm{NMe} ; \mathrm{R}_{4}=\mathrm{OCH}_{3}$ & 120 & 68 \\
\hline 5 & $\mathrm{X}=\mathrm{N} ; \mathrm{R}_{1} / \mathrm{R}_{4}=\mathrm{OCH}_{3}$ & 340 & 83 \\
\hline 6 & $\mathrm{X}=\mathrm{O} ; \mathrm{R}_{3}=\mathrm{OMe}$ & $180\left(140^{\circ} \mathrm{C}\right)$ & 61 \\
\hline 7 & $\mathrm{X}=\mathrm{O} ;$ All $\mathrm{R}=\mathrm{H}$ & $180\left(140^{\circ} \mathrm{C}\right)$ & 57 \\
\hline
\end{tabular}

\section{CONCLUSION}

The present study has developed a new methodology for oxidative cyclization via functionalization of $\mathrm{C}-\mathrm{H}$ bonds in anilino- and phenyloxy- naphthoquinones to give benzocarbazolquinones and benzonaphthofurandiones in short reaction times and in good yields.

\section{ACKNOWLEDGEMENTS}

PGQU-IQ/UFRJ, CAPES, CNPq, and FAPERJ

\section{REFERENCES}

1. a) Thomson, R. H.; Editor Naturally Occurring Quinones IV: Recent Advances, 4th Edition; Blackie: London, UK, 1997. b) Macias-Ruvalcaba, N., Cuevas, G., González, I., Aguilar-Martínez, M. J. Org. Chem. 2002, 67, 3673. c) Jakeman, D. L.; Farrell, S.; Young, W.; Doucet, R. J.; Timmons, S. C. Bioorg. Med. Chem. Lett. 2005, 15, 1447-9. d) del Corral, J. M. M. Castro, M. A., Gordaliza, M., Martín, M. L., Gamito, A. M., Cuevas, C., Feliciano, A. S. Bioorg. Med. Chem. 2006, 14, 2816.

2. Lisboa, C. S.; Santos, V. G.; Vaz, B. G.; de Lucas, N. C.; Eberlin, M. N. Garden, S. J. J. Org. Chem. 2011, 76, 5264.

3. For example see: a) Echavarren, A. M., Tamayo, N., Paredes, C. Tetrahedron Lett. 1993, 34, 4713. b) Knoelker, H.-J., O'Sullivan, N Tetrahedron 1994, 50, 10893. c) Hagelin, H., Oslob, J. D., Akermark, B. Chem. Eur. J. 1999, 5, 2413. d) Liégault, B., Lee, D., Huestis, M. P., Stuart D. R., Fagnou, K. J. Org. Chem. 2008, 73, 5022. 